\title{
ARTí́CUlO
}

\section{Cambios en la dieta de dos especies de mojarras (Gerreidae) en la Laguna de Términos, Campeche, México}

Diet changes in two species of mojarras (Gerreidae) in Laguna de Términos, Campeche, Mexico

\section{Alma Pineda-Peralta ${ }^{1}$, Julia Ramos-Miranda ${ }^{2}$, Manuel Castillo-Rivera $^{3 *}$ y Francisco Arreguín-Sánchez ${ }^{4}$}

\author{
'Doctorado en Ciencias Biológicas y de la Salud, Universidad Autónoma Metropolitana, Avenida San Rafael Atlixco 186, Colonia \\ Vicentina, CP 09340, México, D.F., México \\ ${ }^{2}$ Instituto de Ecología, Pesquerías y Oceanografía del Golfo de México (EPOMEX), Laboratorio de Pesquerías, Universidad Autónoma \\ de Campeche, Av. Agustín Melgar s/n, entre Juan de la Barrera y calle 20, Col. Buenavista, CP 24039, Campeche, México \\ ${ }^{3}$ Departamento de Biología, Laboratorio de Peces, Universidad Autónoma Metropolitana, Unidad Iztapalapa, Avenida San Rafael \\ Atlixco 186, Colonia Vicentina, CP09340, México, D.F., México. *crma@xanum.uam.mx \\ ${ }^{4}$ Centro Interdisciplinario de Ciencias Marinas (CICIMAR), Instituto Politécnico Nacional, Av. Instituto Politécnico Nacional s/n Col., \\ Playa Palo de Santa Rita, CP 23096, La Paz, B.C.S., México
}

Abstract.- The mojarras Diapterus rhombeus and Eucinostomus gula are nektonic dominant species and play an essential role as a link in the energy transfer in the Terminos Lagoon. Thus, in order to contribute on the knowledge of their trophic dynamics, we analyzed the inter-specific and ontogenetic (intra-specific) variation in the diet of these species. A total of 156 individuals of $D$. rhombeus were analyzed, which consumed 16 trophic groups, whereas 134 individuals of E. gula were analyzed and these consumed 13 trophic groups. The relative importance of trophic groups (gravimetric method) showed that the overall diet of both species was determined mainly by Detritus and Algae, with slight differences between species. The asymptotic pattern of the cumulative trophic diversity indicates that the diets are accurately represented for the study area. Ontogenetically (intra-specific level), the diet of smaller individuals of D. rhombeus (with Copepoda as main food source) showed significant differences with the diets of larger individuals; whereas the diets of larger individuals showed no difference among them. Also, there was a trend in which similarity in diet was lower when the separation in the size of fish was greater, indicating a trend in decreasing intraspecific competition. In contrast, for E. gula, there were no significant differences between the size groups analyzed. Therefore, for $D$. rhombeus the inter-specific competition for food resources may become stronger than intra-specific competition, while for $E$. gula, the intra-specific competition for food resources is stronger than interspecific competition.

Key words: Trophic resources, competition, resource partitioning, differential consumption, trophic similarity

Resumen.- Las mojarras Diapterus rhombeus y Eucinostomus gula son especies dominantes del necton y contribuyen a la transferencia energética en la Laguna de Términos. Así, con el fin de contribuir al conocimiento sobre su dinámica trófica, se evaluó la variación inter-específica e intra-específica en sus dietas. Para D. rhombeus se analizaron 156 individuos, identificándose 16 ítems alimentarios; para E. gula se analizaron 134 individuos, consumiendo estos 13 ítems alimentarios. Las importancias relativas de los ítems alimentarios (método gravimétrico) mostraron que la dieta general de ambas especies estuvo determinada principalmente por Detritus y Algae, existiendo ligeras diferencias entre especies. El comportamiento asintótico de la diversidad trófica acumulada indica que las dietas están idóneamente representadas para el área de estudio. A nivel ontogénico (intra-específico), la dieta de los organismos pequeños de D. rhombeus (con Copepoda como una fuente importante de alimento), mostró diferencias significativas con las dietas de los individuos grandes; mientras que las dietas de los organismos grandes no mostraron diferencias entre sí. Se observó una tendencia a que la similitud de las dietas fuera menor cuando existe una mayor separación en el tamaño de los peces, indicando una tendencia en la disminución de la competencia intra-específica. Por el contrario, para E. gula las dietas de los grupos de tamaño analizados no mostraron diferencias significativas. De acuerdo con estos resultados, para $D$. rhombeus la competencia inter-específica por recursos tróficos llegaría a ser más fuerte que la competencia intra-específica, mientras que para E. gula, la competencia intra-especifica podría ser más fuerte que la inter-especifica.

Palabras clave: Recursos tróficos, competencia, partición de recursos, consumo discriminado, similitud trófica 


\section{INTRODUCCIÓN}

El principal objetivo de la ecología trófica de peces es entender su historia natural y su papel en la dinámica trófica de los ecosistemas acuáticos. Así, el conocimiento de la ecología trófica contribuye al entendimiento de las de interacciones tróficas, nicho trófico, patrones de partición de recursos, competencia y transferencia de energía (Blaber 2000, Braga et al. 2012, Specziár \& Erõs 2014). La partición de recursos se entiende como cualquier diferencia substancial en el uso de los recursos (alimento y espacio), y aunque estas diferencias pueden ser debidas a factores ambientales y conductuales, el objetivo principal de la mayoría de los estudios sobre este tema es describir el papel que juega la competencia en los procesos de diferenciación de nicho, que permiten a las especies coexistir establemente (Schoener 1974), regulando de esta manera la estructura de las comunidades de peces (Ross 1986, Gerking 1994, Scott et al. 2007).

Los patrones de partición de recursos han recibido mayor atención al nivel de la competencia inter-específica, pero el mismo proceso también puede ocurrir dentro de una especie (Gerking 1994, Alanärä et al. 2001), por los que en muchos estudios, los efectos inter- e intra-específicos se han abordado simultáneamente (Jones 2002, Scott et al. 2002, Specziár \& Erõs 2014). En este sentido, en la mayoría de las especies de peces de la clase Actinopterygii se han observado cambios en los hábitos de alimento a lo largo de su ontogenia (Wootton 1990, Blaber 2000, Jones 2002, Specziár \& Erõs 2014), lo cual está relacionado con los cambios de hábitat (Machias \& Labropoulou 2002), las limitaciones morfológicas (Labropoulou \& Eleftheriou 1997), requerimientos energéticos (Lilly \& Fleming 1981) y la capacidad de asimilar cierto tipo de presas (Molinero \& Flos 1991). De este modo, los peces reparten el recurso trófico alimentándose de presas diferentes o aprovechando el mismo recurso en diferentes etapas de su ciclo de vida. Asimismo, organismos diferentes tienden bajo presiones ambientales equivalentes, a desarrollar características semejantes que les permiten explotar recursos similares, generándose así los gremios tróficos (Root 1967).

Las mojarras participan en la trama trófica como eslabón de la energía entre los consumidores primarios y piscívoros superiores (Aguirre-León et al. 1982, Santos \& Araújo 1997). Particularmente, estas especies son explotados a mediana escala para el consumo humano y son un recurso pesquero relevante de tipo artesanal (Castillo-Rivera et al. 2005). El conocimiento de los hábitos de alimento de las mojarras es importante debido a que por su abundancia desempeñan una función crucial en la transferencia, intercambio y almacenamiento de energía dentro y fuera de los sistemas costeros. Así, a partir de esta información es posible realizar un manejo óptimo de los recursos pesqueros, ya que forman parte de un sistema multiespecífico, en donde las interacciones depredador-presa influyen directamente en las pesquerías (Pauly 1986).

La Sonda de Campeche frente a la Laguna de Términos es considerada una de las zonas pesqueras más importante en México, con una vasta variedad de recursos pesqueros. Particularmente en la Laguna de Términos, en numerosos estudios se señala que la comunidad íctica está integrada por cerca de 100 especies, de las cuales 15 se consideran dominantes (Amezcua-Linares \& Yáñez-Arancibia 1980, Yáñez-Arancibia \& Day 1982, Ayala-Pérez et al. 1993, Ramos-Miranda et al. 2005). Dentro del sistema, las mojarras Diapterus rhombeus (Cuvier, 1829) y Eucinostomus gula (Quoy \& Gaimard, 1824) han mostrado ser unas de las especies dominantes en número, peso y frecuencia de captura.

Aunque en el área de estudio existen trabajos que describen la dieta de las mojarras D. rhombeus y E. gula (Aguirre-León \& Yáñez-Arancibia 1986, Aguirre-León \& Díaz-Ruiz 2006), pocos analizan simultáneamente el uso diferencial de los recursos tróficos a nivel inter e intra-específico. En este sentido y considerando los modelos de la teoría de competencia (Morin 2011), se estableció la hipótesis que los cambios intraespecíficos en la dieta mostrarán diferencias substanciales con respecto a los cambios inter-específicos, para las especies Diapterus rhombeus y Eucinostomus gula. El objetivo del presente trabajo fue determinar de qué manera se reparte el recurso trófico entre $D$. rhombeus y E. gula en la Laguna de Términos, Campeche, así como los cambios ontogénicos en la dieta de cada especie.

\section{Materiales Y MÉTODOS}

La Laguna de Términos se localiza entre $91^{\circ} 10^{\prime}-92^{\circ} 00^{\prime} \mathrm{O}$ y $18^{\circ} 20^{\prime}-19^{\circ} 00^{\prime} \mathrm{N}$ en el estado mexicano de Campeche, al sur del Golfo de México. De noviembre 2010 a octubre 2011 se realizaron mensualmente campañas de campo en 17 sitios de muestreo distribuidos en el sistema Laguna de Términos (Fig. 1).

Se registró la longitud ( $0,1 \mathrm{~cm}$ de precisión) y el peso total (g) de cada individuo con una balanza digital (Ohaus $0,1 \mathrm{~g}$ de precisión). Los organismos fueron diseccionados y los estómagos extraídos, conservándose en formol al 5\%. Posteriormente para analizar la dieta de los organismos, los estómagos se lavaron y se extrajo el contenido estomacal, el cual fue distribuido sobre cajas de Petri y examinado bajo un microscopio estereoscópico (40x). De acuerdo con las posibilidades de identificación, el material examinado se 


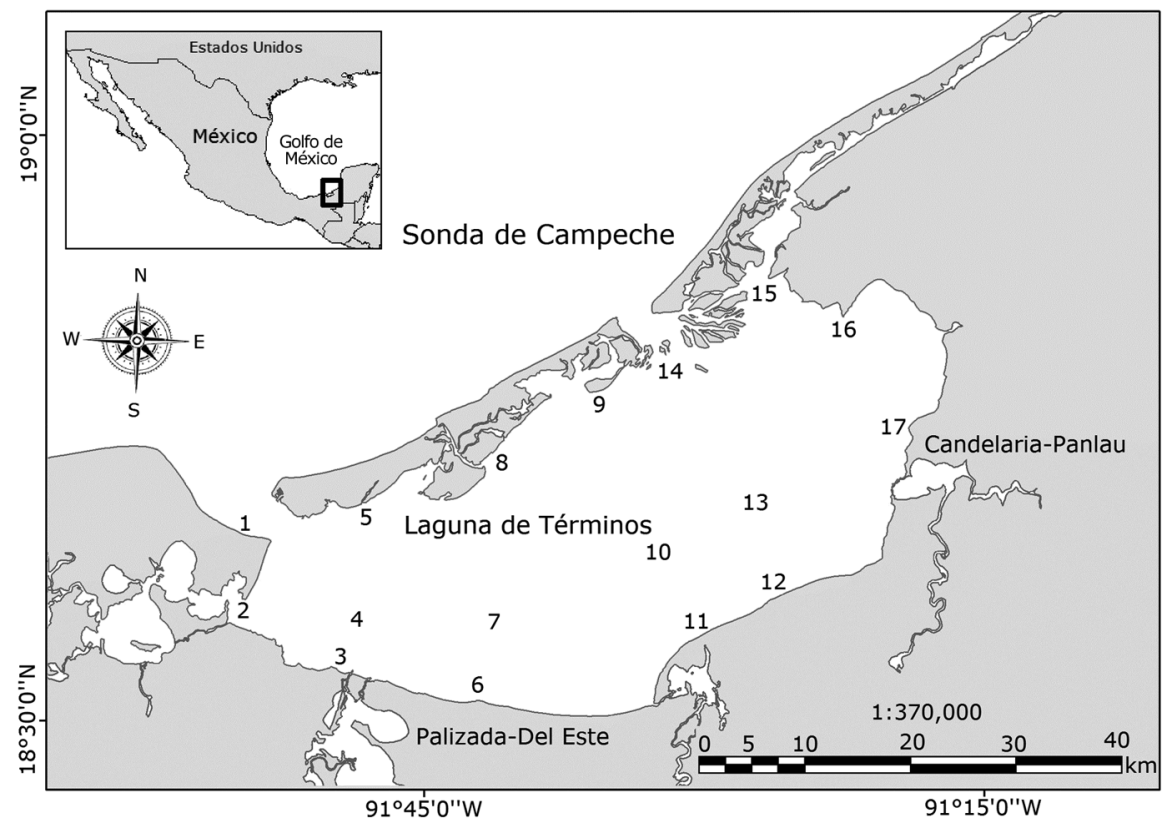

Figura 1. Localización geográfica de la Laguna de Términos y sitios de muestreo / Geographical location of Terminos Lagoon and sampling sites

categorizó en ítems alimentarios utilizando literatura especializada. La importancia relativa (IR) de cada ítem alimentario se determinó mediante el método gravimétrico utilizando una balanza analítica con precisión de 0,00001 g y fue expresada como un porcentaje. Asimismo, para la dieta general se determinó también la frecuencia de aparición (FA) con la que cada especie consumió un determinado ítem, expresándose como el porcentaje de estómagos donde apareció determinada presa, con respecto al número total de estómagos analizados (Hyslop 1980). Debido a que la variación ontogénica es casi universal en peces (Stoner \& Livingston 1984), para analizar esta variación en la dieta de las especies, se establecieron grupos de tallas con una amplitud suficiente para detectar cambios mínimos en la dieta (Castillo-Rivera 2011), en los cuales quedaron incluidos los individuos de talla mínima y máxima de ambas especies. Así, se definieron 4 grupos de longitud de 4,0 cm cada uno: Clase $1(2,05-6,05 \mathrm{~cm})$, Clase $2(6,05-10,05 \mathrm{~cm})$, Clase $3(10,05-14,05 \mathrm{~cm})$, Clase $4(14,05-$ $18,05 \mathrm{~cm})$.

\section{AnÁlisis de DATOS}

Para evaluar si el número de contenidos estomacales fue suficiente para describir adecuadamente la dieta de las especies en el área estudiada, se utilizó la metodología propuesta por Hoffman (1978), que consiste en graficar la diversidad trófica
(H') acumulada al i-ésimo estómago revisado, la cual si llega a alcanzar la asíntota, indicará que el número de muestras analizadas fue suficiente. Se consideró que la asíntota se alcanza, cuando el coeficiente de variación de la diversidad trófica acumulada presenta un valor $\leq 0,05$, lo que implica un nivel de confianza del $95 \%$ en la determinación de la variabilidad de las dietas. La diversidad trófica se estimó con el índice de ShannonWiener utilizando el paquete EstimateS (Colwell 2013). Este índice también se utilizó para determinar la amplitud de nicho trófico de cada individuo (Krebs 1999), la cual fue empleada para definir el tipo de estrategia alimentaria de los peces (Especialista-Generalista).

Con el objetivo de determinar si existían diferencias estadísticamente significativas entre la dieta de dos grupos de peces y para identificar los ítems alimentarios más significativos en la diferenciación de estas dietas, se aplicaron Análisis de Discriminantes. Como variables discriminatorias se utilizaron las importancias relativas (derivadas del método gravimétrico) de cada uno de los ítems alimentarios y las variables clasificatorias fueron las especies y grupos de talla. Para medir las similitudes en las dietas se utilizó el criterio de Lambda de Wilks $(\lambda)$, la cual es tan fácil de interpretar como un índice de similitud o sobreposición de nicho, porque sus valores varían de 1 (total similitud entre grupos) a 0 (total diferencia entre grupos). Finalmente, para evaluar la importancia que tiene cada 
ítem alimentario en la separación de la dieta se utilizó la matriz de correlación, la cual está constituida por las correlaciones entre las variables discriminatorias originales y las correspondientes funciones discriminantes derivadas, por lo que, entre más grande es cada coeficiente de correlación, más grande es la importancia de un ítem alimentario particular en la diferenciación de las dietas (Castillo-Rivera et al. 2000). Aunque métodos más sencillos han sido utilizados para evaluar las diferencias o similitudes en la composición de la dieta de los peces, como los índices de similitud o sobreposición de nicho (Krebs 1999), estos índices tienen el problema que son coeficientes descriptivos y no estimadores de algún parámetro estadístico que permita pruebas de significación o la definición de intervalos de confianza fiables (Ludwig \& Reynolds 1988, Krebs 1999). Además, estos métodos son bivariados y la comparación de las dietas de los peces es por sí misma un caso multivariado, porque se comparan muchos peces y cada pez consume varias presas. Por esta razón, un análisis multivariado como el análisis de discriminantes, permite una adecuada aproximación para evaluar similitudes o diferencias entre la dieta de grupos de peces, por lo que se le ha utilizado con frecuencia en muchos estudios de este tipo (Labropoulou \& Eleftheriou 1997, Castillo-Rivera et al. 2000, Jones 2002, Scott et al. 2007, Giller \& Greenberg 2015). En el presente estudio, este análisis fue desarrollado utilizando el paquete SPSS $(2008)^{1}$.

\section{Resultados}

\section{Dieta General}

De Diapterus rhombeus se capturó un total de 550 individuos, mientras que para Eucinostomus gula se capturaron 423. De acuerdo con el estado de conservación de los peces y tratando de tener representados todos los intervalos de tamaño, sitios de muestreo y meses de colecta, para el análisis de contenido estomacal se eligieron al azar 156 individuos de la primera especie, con un intervalo de tallas de 3,9 a $17,3 \mathrm{~cm}$, registrándose 5 estómagos vacíos. Para la segunda especie se eligieron al azar 134 individuos, con una variación de tamaño de 6,1 a $12,3 \mathrm{~cm}$, de los cuales 14 se encontraron con estómagos vacíos.

En general, la dieta de $D$. rhombeus se constituyó de al menos 16 ítems alimentarios, siendo los de mayor frecuencia de consumo: Detritus, Foraminifera, Ostracoda, Algae, Restos Vegetales y Copepoda. El análisis del contenido estomacal indicó que $E$. gula consumió un total de 13 componentes tróficos, siendo el ítem alimentario preferencial el Detritus seguido por Algae, Foraminifera y Ostracoda (Tabla 1).
Tabla 1. Importancia Relativa (IR \%) y Frecuencia de Aparición (FA \%) de los ítems alimentarios encontrados en los contenidos estomacales de las dos especies de mojarras en la Laguna de Términos, México / Relative importance (IR \%) and frequency of occurrence (FA \%) of each trophic group, in the diets of both species of mojarras in the Terminos Lagoon, Mexico

\begin{tabular}{|c|c|c|c|c|}
\hline \multirow{2}{*}{ Categorías tróficas } & \multicolumn{2}{|c|}{ Diapterus rhombeus } & \multicolumn{2}{|c|}{ Eucinostomus gula } \\
\hline & IR & FA & IR & FA \\
\hline Foraminifera & 2,478 & 70,861 & 1,805 & 14,876 \\
\hline Porifera & 0 & 0 & 0,168 & 0,826 \\
\hline Cestoda & 0,064 & 1,325 & 0 & 0 \\
\hline Acanthocephala & 0,001 & 0,662 & 0 & 0 \\
\hline Nematoda & 0,435 & 6,623 & 0,004 & 0,826 \\
\hline Polychaeta & 0 & 0 & 2,860 & 4,132 \\
\hline Ophiuroidea & 0,075 & 0,662 & 0 & 0 \\
\hline Bivalvia & 1,148 & 6,623 & 0 & 0 \\
\hline Gastropoda & 0,667 & 6,623 & 0,350 & 0,826 \\
\hline Ostracoda & 1,228 & 43,709 & 2,669 & 12,397 \\
\hline Copepoda & 3,011 & 25,828 & 0,061 & 4,959 \\
\hline Tanaidacea & 0,605 & 4,636 & 1,204 & 3,306 \\
\hline Amphipoda & 0,021 & 1,325 & 0,016 & 1,653 \\
\hline Decapoda & 0,621 & 1,325 & 0 & 0 \\
\hline Restos de Teleósteos & 0,172 & 1,987 & 0,529 & 4,132 \\
\hline Algae & 19,544 & 35,762 & 23,848 & 35,537 \\
\hline Restos vegetales & 3,801 & 31,788 & 0,350 & 4,132 \\
\hline Detritus & 66,129 & 83,444 & 66,136 & 80,165 \\
\hline
\end{tabular}

Considerando el coeficiente de variación de la diversidad trófica acumulada cuando presenta un valor $\leq 0,05$, las dos especies de mojarras alcanzan una asíntota aproximadamente al $56 \%$ de los estómagos analizados (85 individuos) de $D$. rhombeus y cerca del $43 \%$ de los estómagos de E. gula (53 individuos) (Fig. 2). Por lo anterior, se puede señalar que los hábitos de alimento reportados en el presente estudio, pueden representar fielmente la dieta de las especies en la Laguna de Términos.

\section{ANÁLISIS TRÓfico INTER-ESPECÍFICO}

En términos de importancia relativa (IR) ambas especies consumieron principalmente Detritus y Algae, representando entre ambos ítems alimentarios más del $85 \%$ en cada especie. Una fuente secundaria de alimento para $D$. rhombeus fueron Restos Vegetales, Copepoda y Foraminifera, mientras que para E. gula fueron Polychaeta y Ostracoda (Tabla 1). Aunque existió una similitud relativamente alta $(\lambda=0,854)$ entre la dieta de estas dos especies, el análisis de discriminantes detectó diferencias $(P$ $<0,001)$, las cuales fueron debidas a las variaciones en el consumo de las fuentes secundarias de alimento. Así, los ítems alimentarios que más contribuyeron a las diferencias de las dietas fueron: Copepoda $(0,474)$, Restos Vegetales $(0,444)$ y Polychaeta (-0,326). En relación con la amplitud de nicho trófico, ésta mostró que el promedio de $D$. rhombeus $(\overline{\mathrm{x}}=0,603)$ fue mayor al promedio de E. gula $(\overline{\mathrm{x}}=0,227)$.

${ }^{1}$ SPSS. 2008. SPSS Statistics for Windows, Version 17.0. SPSS, Chicago. 

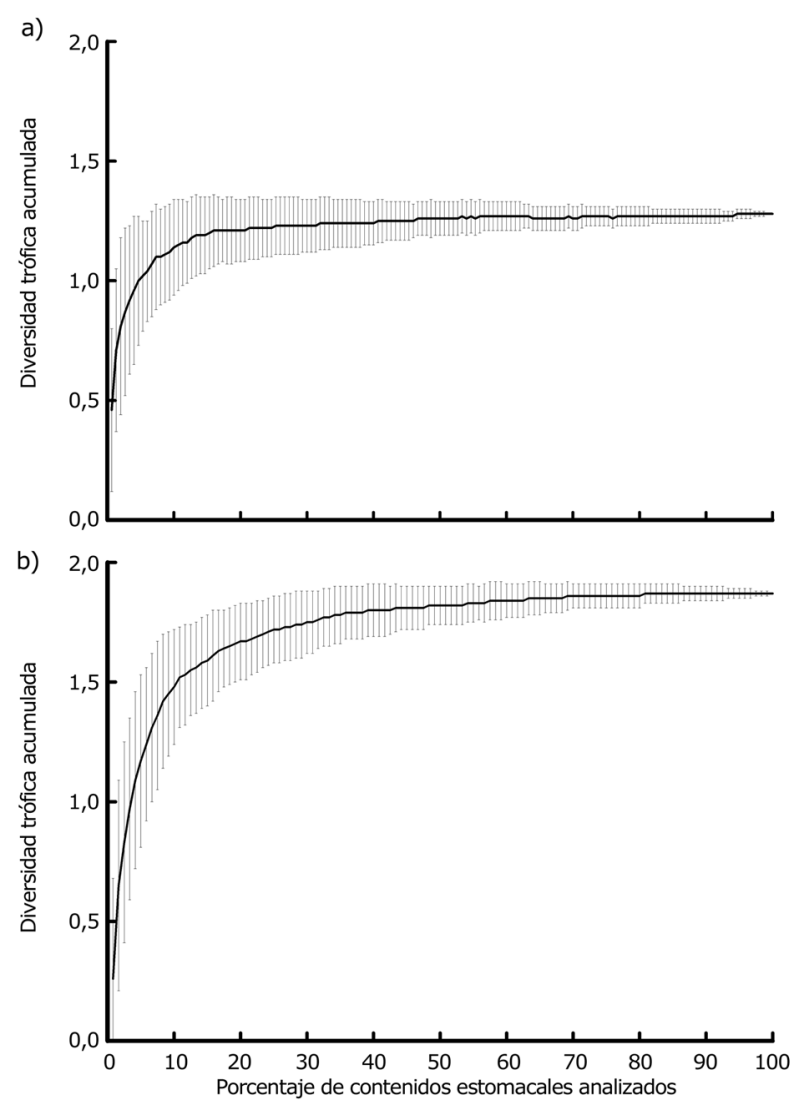

Figura 2. Comportamiento de la diversidad trófica acumulada (promedio y desviación estándar) de acuerdo con el porcentaje de contenidos estomacales analizados secuencialmente para Diapterus rhombeus (a) y Eucinostomus gula (b) / Cumulative trophic diversity (mean and standard deviation) according to the percentage of individuals analyzed sequentially for Diapterus rhombeus (a) and Eucinostomus gula (b)

\section{ANÁLISIS TRÓFICO ONTOGÉNICO (INTRA-ESPECÍfICO)}

El análisis ontogénico de los hábitos de alimento de $D$. rhombeus mostró al detritus como la principal fuente de alimento en todas las tallas, existiendo un ligero incremento en su consumo a medida que los peces crecen. Asimismo, los ítems alimentarios preferidos por las tallas pequeñas fueron Copepoda, Restos Vegetales y Ostracoda. Las tallas intermedias mostraron un incremento en el consumo de Algae, mientras que ítems alimentarios preferenciales para los organismos de tallas mayores fueron Algae, Bivalvia y Tanaidacea (Tabla 2).

Análisis de discriminantes permitieron definir que la dieta de los individuos de tamaño 1 y 2 de D. rhombeus, fueron significativamente diferentes, determinadas por las diferencias en el consumo de Copepoda (-0,693), Restos de Teleósteos $(-0,319)$ y Algae $(0,221)$. La dieta de los organismos de tamaño
Tabla 2. Variación ontogénica de la Importancia Relativa (IR \%) de cada ítem alimentario en la dieta de D. rhombeus en la Laguna de Términos, México / Ontogenetic variation of the Relative Importance (IR \%) of each food item in the diet of D. rhombeus in the Terminos Lagoon, Mexico

\begin{tabular}{|c|c|c|c|c|}
\hline \multirow{2}{*}{ Categorías tróficas } & $\begin{array}{c}\text { Grupos de } \\
\text { talla } 1 \\
\end{array}$ & $\begin{array}{c}\text { Grupos de } \\
\text { talla } 2 \\
\end{array}$ & $\begin{array}{c}\text { Grupos de } \\
\text { talla } 3\end{array}$ & $\begin{array}{c}\text { Grupos de } \\
\text { talla } 4\end{array}$ \\
\hline & $\operatorname{IR}(n=13)$ & $\operatorname{IR}(\mathrm{n}=80)$ & $\operatorname{IR}(\mathrm{n}=39)$ & $\operatorname{IR}(n=19)$ \\
\hline Foraminifera & 1,676 & 3,484 & 1,362 & 1,084 \\
\hline Porifera & 0 & 0 & 0 & 0 \\
\hline Cestoda & 0 & 0 & 0 & 0,496 \\
\hline Acanthocephala & 0 & 0 & 0 & 0 \\
\hline Nematoda & 0 & 0,066 & 0,223 & 2,705 \\
\hline Polychaeta & 0 & 0 & 0 & 0 \\
\hline Ophiuroidea & 0 & 0,138 & 0 & 0 \\
\hline Bivalvia & 0 & 0 & 1,503 & 6,026 \\
\hline Gastropoda & 0 & 0,230 & 1,110 & 2,053 \\
\hline Ostracoda & 2,108 & 1,639 & 0,692 & 0 \\
\hline Copepoda & 22,462 & 1,978 & 0,115 & 0 \\
\hline Tanaidacea & 0 & 0 & 0,038 & 4,726 \\
\hline Amphipoda & 0 & 0 & 0,003 & 0,163 \\
\hline Decapoda & 0 & 0 & 2,397 & 0,016 \\
\hline Restos de Teleósteos & 1,477 & 0,085 & 0 & 0 \\
\hline Algae & 1,738 & 23,844 & 21,585 & 9,437 \\
\hline Restos Vegetales & 3,392 & 5,374 & 2,544 & 0,037 \\
\hline Detritus & 67,146 & 63,163 & 68,397 & 73,268 \\
\hline
\end{tabular}

1 también difirió significativamente de la alimentación de los individuos de las tallas 3 y 4 , siendo para estas tallas el ítem más importante en la separación de las dietas Copepoda $(-0,750 ; 0,442)$. Estos análisis también mostraron valores de $\lambda$ relativamente bajos, los cuales tendieron a disminuir a medida que difiere más la talla entre los peces, lo cual implica una atenuación en los potenciales procesos de competencia intraespecífica. Similarmente los organismos de tamaño 2 mostraron diferencias significativas con los organismos de tamaño 3 y 4 determinadas principalmente por el consumo de Bivalvia $(0,514 ;-0,432)$ y Foraminifera $(-0,496 ; 0,301)$. Al igual de como sucedió con los peces de tamaño 1 , los valores de $\lambda$ tendieron a disminuir entre los peces que se encuentran más separados en tamaño. Finalmente la alimentación de los organismos de tamaño 3 y 4 no mostraron diferencias significativas (Tabla 3). En relación con la amplitud de nicho trófico, para D. rhombeus los promedios más altos los mostraron los individuos de los grupos de tamaño $1(\overline{\mathrm{x}}=0,933)$ y tamaño $2(\bar{x}=0,633)$, mientras que los valores más bajos correspondieron a los individuos de tamaño $3(\overline{\mathrm{x}}=0,464)$ y tamaño $4(\bar{x}=0,537)$.

La Tabla 4 muestra el patrón de la alimentación por tallas de E. gula, el cual estuvo determinado principalmente por el 
Tabla 3. Resultados del análisis de discriminantes por grupos de talla, aplicados a la dieta de Diapterus rhombeus / Results of discriminant analysis by size classes, applied to Diapterus rhombeus diets

\begin{tabular}{lccc}
\hline & $\begin{array}{c}\text { Grupos de } \\
\text { talla 1 }\end{array}$ & $\begin{array}{c}\text { Grupos de } \\
\text { talla 2 }\end{array}$ & $\begin{array}{c}\text { Grupos de } \\
\text { talla 3 }\end{array}$ \\
\hline Grupos de talla 2 & $\lambda=0,498$ & & \\
& $P<0,0001$ & & \\
Grupos de talla 3 & $\lambda=0,437$ & $\lambda=0,802$ & \\
& $P<0,0001$ & $P=0,027$ & \\
Grupos de talla 4 & $\lambda=0,252$ & $\lambda=0,624$ & $\lambda=0,733$ \\
& $P=0,001$ & $P<0,0001$ & $P=0,214$ \\
\hline
\end{tabular}

Tabla 4. Variación ontogénica de la Importancia Relativa (IR \%) de cada ítem alimentario en la dieta de Eucinostomus gula en la Laguna de Términos, México / Ontogenetic variation of the Relative Importance (IR \%) of each food item in the diet of Eucinostomus gula in the Terminos Lagoon, Mexico

\begin{tabular}{lcc}
\hline Categorías tróficas & $\begin{array}{c}\text { Grupos de } \\
\text { talla } 2 \\
\text { IR }(\mathrm{n}=109)\end{array}$ & $\begin{array}{c}\text { Grupos de } \\
\text { talla } 3 \\
\text { IR }(\mathrm{n}=11)\end{array}$ \\
\hline Foraminifera & 1,993 & 0 \\
Porifera & 0,186 & 0 \\
Cestoda & 0 & 0 \\
Acanthocephala & 0 & 0 \\
Nematoda & 0,005 & 0 \\
Polychaeta & 3,174 & 0 \\
Ophiuroidea & 0 & 0 \\
Bivalvia & 0 & 0 \\
Gastropoda & 0,389 & 0 \\
Ostracoda & 2,950 & 0 \\
Copepoda & 0,068 & 0 \\
Tanaidacea & 1,337 & 0 \\
Amphipoda & 0,017 & 0 \\
Decapoda & 0 & 0 \\
Restos de Teleósteos & 0,587 & 0 \\
Algae & 23,058 & 33,845 \\
Restos Vegetales & 0,302 & 0,855 \\
Detritus & 65,936 & 65,300 \\
\hline
\end{tabular}

consumo de Detritus y Algae (> 88\%) para ambos grupos de tamaño. Así, el análisis discriminante mostró que para $E$. gula, no existen diferencias significativas entre estos grupos de tamaño $(P=0,981)$, mostrando una fuerte similitud en los recursos tróficos consumidos $(\lambda=0,969)$. Para esta especie, el promedio de amplitud trófica de los individuos de tamaño $2(\overline{\mathrm{x}}=0,234)$ fue mayor al de los individuos de tamaño $3(\overline{\mathrm{x}}=0,112)$.

\section{Discusión}

Los Gerreidos son peces eurihalinos de gran importancia económica y ecológica que cumplen una función trófica definida en las redes alimentarias de los ecosistemas lagunares costeros (Aguirre-León et al. 1982). La disponibilidad de información sobre la alimentación de peces estuarinos, como en el presente caso, frecuentemente es de vital importancia para propósitos de manejo de pesquerías y conservación. Sin un detallado conocimiento de los complejos requerimientos de alimento, patrones de conducta trófica y relaciones presa-depredador, no es posible describir cómo las comunidades de peces estuarinos funcionan, ni es posible interpretar o predecir cambios que podrían resultar de cualquier intervención natural o antropogénica (Blaber 2000, Braga et al. 2012).

La composición de la dieta de las especies analizadas muestra que los principales ítems alimentarios fueron Detritus y Algae (IR > 85\%), consumiendo eventualmente pequeños invertebrados. Resultados similares han sido reportados históricamente por otros autores (Randall 1967, Furtado 1969, Odum \& Heald 1972, Carr \& Adams 1973, González-Sansón \& Rodríguez-Viñas 1983, Kerschner et al. 1985, Rivas et al. 1999), lo que representa una constancia en la conducta trófica de la familia. Particularmente para la laguna de Términos, la dieta de $D$. rhombeus fue analizada en las décadas de los ' 80 s (Aguirre-León \& Yáñez-Arancibia 1986) y ‘90s (Aguirre-León \& Díaz-Ruíz 2006). En ambos períodos, al igual que el presente estudio, el Detritus fue la principal fuente de alimento de esta especie, sin embargo, el consumo de otros recursos tróficos ha variado a lo largo de estos períodos. Así, se observa que ha existido un progresivo incremento en el consumo de recursos vegetales y una disminución en el consumo de Polychaeta. Similarmente para el caso de E. gula, también ha existido una disminución en el consumo de Polychaeta (la principal fuente de alimento en los ' 80 s) y un incremento en el consumo de Detritus y componentes vegetales (presente estudio). A pesar que estas especies se alimentan de organismos del fondo, no se encontró evidencia del consumo de diatomeas bentónicas.

Las preferencias en la dieta de estas especies se pueden relacionar con el hecho que a la Laguna de Términos se asocian una importante vegetación (mangle y pastos marinos) y ríos, lo 
que la convierte en una zona de almacenamiento y transporte de materia orgánica, sedimentos, detritos y organismos (microcrustáceos), convirtiendo estas fuentes potenciales de alimento en más recursos tróficos para un mayor número de peces, lo cual se ve reflejado primariamente en las altas abundancia de juveniles en esta zona. En este sentido Yáñez-Arancibia \& Sánchez-Gil (1983) mencionan que las características ecológicas en la Laguna de Términos tales como circulación litoral, intercambio de aguas oceánicas y costeras, descarga fluvial y transición de material terrígeno y de sedimentos calcáreos, condicionan un sistema sedimentario y comunidades biológicas dominadas por organismos detritívoros. De acuerdo a Gerking (1994), es realmente difícil establecer cuando una especie es detritívora, sin embargo, un elevado consumo de detritus, como es el caso de las especies en estudio, hace que se sitúe a las mojarras en este nivel trófico.

Randall (1967) indica que las mojarras son principalmente consumidores bentónicos, con una boca muy retráctil que les permite alimentarse de invertebrados de la infauna. De acuerdo con Andreata (1988), la morfología de estos organismos es considerada de las más simples entre los perciformes, sin embargo, sobresale su influencia en el mecanismo de proyección bucal durante la alimentación (Schaeffer \& Rosen 1961, Gosline 1981, Motta 1984). Esta proyección se debe al desarrollo del premaxilar el cual genera una alta capacidad retráctil de la boca que caracteriza a la familia en general (Andreata \& Barbiéri 1981, Andreata 1988, Kobelkowsky \& Alemán-Rivero 2000), proporcionando el mecanismo de alcance para las presas del componente bentónico que conforman su alimento preferencial (Cyrus \& Blaber 1982).

Existe un elevado número de especies coexistentes que utilizan recursos comunes y que pueden relacionarse por su afinidad en la utilización de éstos, formando asociaciones o gremios como resultado de esta superposición trófica (Ludwig \& Reynolds 1988). Tal es el caso de Diapterus rhombeus y Eucinostomus gula, las cuales de acuerdo con los valores de $\lambda$ mostraron cierto grado de similitud en la dieta (Tabla 3), dejando ver para ambas especies la preferencia por el consumo de Detritus y Algae. En este sentido, de acuerdo con el concepto de nicho, diferencias entre especies facilitan su co-existencia a largo plazo, aunque en un período de corto tiempo, especialmente cuando uno o más recursos alimentarios son abundantes, las similitudes dietéticas inter-especificas se pueden incrementar (Specziár \& Erõs 2014).

A pesar que ambas especies centraron su alimentación en Detritus y Algae, el número de ítems alimentarios consumidos, así como la amplitud nicho trófico, fueron mayores en $D$. rhombeus que en E. gula. Esto implica que D. rhombeus tiende a presentar una estrategia alimentaria más generalista, mientras que E. gula presenta una estrategia alimentaria más especializada.

Otro tema relacionado con los hábitos alimenticios y que se ha presentado como un fenómeno generalizado de peces, es el cambio ontogénico en las preferencias alimentarias. A medida que los peces crecen, estos cambian su tamaño por lo que también modifican el tamaño de las mandíbulas, hábitos y velocidad de desplazamiento, lo que se traduce en un cambio sustancial de su alimentación (Wootton 1990, Blaber 2000, Jones 2002, Specziár \& Erõs 2014).

El análisis de los hábitos de alimento para Diapterus rhombeus deja ver un cambio significativo durante su ontogenia, debido a que cuando los organismos aumentan de talla existe un incremento progresivo en la ingesta de Detritus y un decremento de organismos pequeños, principalmente de Copepoda. De acuerdo con los valores de $\lambda$, las mayores diferencias se detectaron entre el grupo de tamaño más pequeño y los más grandes (tallas 3 y 4), mientras que las mayores similitudes se observaron entre los individuos medianos y grandes. Así, los valores de $\lambda$ tendieron a disminuir a medida que la segregación por tamaño de los peces es mayor, lo que podría permitir una atenuación de la competencia intra-específica por recursos tróficos, como lo señalan Specziár \& Erõs (2014).

Estas variaciones en la conducta trófica con respecto a su crecimiento pueden deberse en gran medida a la accesibilidad del recurso (Guevara et al. 2007), a los cambios estacionales (Cruz-Escalona et al. 2005) y a los cambios existentes en la morfología, los cuales se correlacionan con el movimiento (forma del cuerpo), dentición, dimensiones de la boca y del intestino del pez (Stoner \& Livingston 1984).

Por el contrario, de acuerdo con los resultados obtenidos en el análisis discriminante para Eucinostomus gula, no existieron diferencias significativas entre tallas, basándose la dieta para ambas tallas en Detritus, Algae y Restos vegetales.

En ambas especies se observó una disminución en la amplitud de nicho trófica a medida que los peces aumentan de talla, por lo que en términos de estrategia alimentaria, los organismos tienden a ser más generalistas cuando son pequeños, adquiriendo una estrategia más especialista cuando alcanzan tallas mayores. Esta disminución en la amplitud de nicho trófico parece ser un efecto común para muchas especies de peces (Castillo-Rivera et al. 2000, Scharf et al. 2000).

Considerando el grado de similitud de las dietas, todos los valores de $\lambda$ del análisis ontogénico (nivel intra-específico) de Diapterus rhombeus (Tabla 3 ), fueron menores al valor de $\lambda$ mostrado en el análisis entre especies $(0,854)$. Lo anterior 
implica, que para esta especie la competencia inter-específica por recursos tróficos podría llegar a ser más fuerte que la competencia intra-específica. Por el contrario, para Eucinostomus gula, el valor de $\lambda$ del análisis entre tamaños $(0,992)$, fue mayor que el valor de $\lambda$ derivado del análisis entre especies, por lo que se puede considerar que para esta especie, la competencia intra-específica por recursos tróficos podría llegar a ser más fuerte que la competencia inter-específica.

Indudablemente los procesos de competencia entre especies similares y sus respuestas a través de la partición de recursos, no se dan solo en el eje de los recursos alimentarios, ya que los patrones de segregación espacial y temporal, también juegan un papel importante en estas interacciones ecológicas. Por lo anterior resulta evidente que otras líneas de investigación quedan abiertas, como aquellas que analicen los patrones espaciotemporales de segregación entre estas especies, así como también aquellas que evalúen las diferencias morfológicas (morfología funcional) que contribuyan a explicar con más detalle los resultados observados en el presente estudio.

\section{Agradecimientos}

Al doctorado en Ciencias Biológicas y de la Salud de la Universidad Autónoma Metropolitana; así como al grupo de trabajo que apoyó en las actividades de campo y laboratorio del Instituto EPOMEX de la Universidad Autónoma de Campeche. La presente investigación forma parte de la tesis para obtener el grado de Doctora en Ciencias Biológicas y de la Salud de Alma Delia Pineda Peralta y se realizó por medio del proyecto financiado por CONACYT (México) 'Long-term effects of environmental changes on the nekton biodiversity and the functioning of tropical estuaries', (México-Francia, Modalidad ANR, C0004-2009-01-000000000111465. 2010-2013). Asimismo se agradecen los valiosos comentarios de dos revisores anónimos, los cuales enriquecieron de manera importante el manuscrito.

\section{LITERATURA CITADA}

Aguirre-León A \& S Díaz-Ruiz. 2006. Estructura de tallas, madurez gonádica y alimentación de Diapterus rhombeus (Gerreidae) en el sistema fluvio-deltaico Pom-Atasta, Campeche, México. Revista de Biología Tropical 54: 599-611.

Aguirre-León A \& A Yáñez-Arancibia. 1986. Las mojarras de la Laguna de Términos: taxonomía, biología, ecología y dinámica trófica (Pisces: Gerreidae). Anales del Instituto de Ciencias del Mar y Limnología, México 13: 369-444.

Aguirre-León A, A Yáñez-Arancibia \& F Amezcua Linares. 1982. Taxonomía, diversidad, distribución y abundancia de las mojarras de la Laguna de Términos Campeche (Pisces: Gerreidae). Anales del Instituto de Ciencias del Mar y Limnología, México 9:213-250.
Alanärä A, MD Burns \& NB Metcalfe. 2001. Intraspecific resource partitioning in brown trout: the temporal distribution of foraging is determined by social Rank. Journal of Animal Ecology 70: 980-986.

Amezcua-Linares F \& A Yáñez-Arancibia. 1980. Ecología de los sistemas fluvio-lagunares asociados a la Laguna de Términos: el hábitat y estructura de las comunidades de peces. Anales del Instituto de Ciencias del Mar y Limnología, UNAM, México 7: 68-118.

Andreata JV. 1988. Consideraçöes sobre a osteologia cefálica do Género Diapterus ranzani, 1840 (Pisces, Perciformes, Gerreidae). Acta Biologica Leopoldensia, Brasil 10: 183-222.

Andreata JV \& LRR Barbiéri. 1981. Osteologia do crânio de Diapterus brasilianus (Cuvier 1830) (Perciformes, Percoidei, Gerreidae). Revista Brasileira de Biologia, Brasil 41:565-574.

Ayala-Pérez LA, A Aguirre-León, OA Avilés-Alatriste, MT Barreiro-Güemes \& JL Rojas-Galaviz. 1993. Peces de sistemas fluvio-lagunares, Laguna de Términos, Campeche. En: Salazar-Vallejo S \& NE González (eds). Biodiversidad marina y costera de México, pp. 596-608. Comisión Nacional de Biodiversidad y CIQRO, México.

Blaber SJM. 2000. Tropical estuarine fishes: Ecology, explotation and conservation, 372 pp. Blackwell Science, Oxford.

Braga RR, H Bornatowski \& JRS Vitule. 2012. Feeding ecology of ûshes: an overview of worldwide publications. Reviews in Fish Biology and Fisheries 22: 915-929.

Carr WES \& CA Adams. 1973. Food habits of juvenile marine fishes occupying seagrass beds in the estuarine zone near Crystal River, Florida. Transactions of the American Fisheries Society 102: 511-539.

Castillo-Rivera M. 2011. Biología trófica de peces costeros. Estuarios del Golfo de México Occidental, 228 pp. Editorial Académica Española, Saarbrücken.

Castillo-Rivera M, A Kobelkowsky \& AM Chávez. 2000. Feeding biology of the flatfish Citharichthys spilopterus (Bothidae) in a tropical estuary of Mexico. Journal of Applied Ichthyology 16: 73-78.

Castillo-Rivera M, M Montiel, L Sanvicente-Añorve \& R Zárate. 2005. Spatial, seasonal and diel distribution patterns of two species of mojarras (Pisces: Gerreidae) in a Mexican tropical coastal lagoon. Journal of Applied Ichthyology 21: 498-503.

Colwell RK. 2013. EstimateS: Statistical estimation of species richness and shared species from samples. Version 9. User's Guide and application. <http://purl.oclc.org/estimates>

Cruz-Escalona VH, MS Peterson, L Campos-Davilla \& M Zetina-Rejón. 2005. Feeding habits and trophic morphology of Inshore Lizardfish (Synodus foetens) on the central continental shelf off Veracruz, Gulf of Mexico. Journal of Applied Ichthyology 21:525-530. 
Cyrus DP \& SJM Blaber. 1982. Mouthpart structure and function and the feeding mechanisms of Gerres (Teleostei). South African Journal of Zoology 17: 117-121.

Furtado E. 1969. Alimentaçao de peixes en aguas estuarinas do estado do Ceará. Arquivos de Ciências do Mar, Brasil 9: 111-114.

Gerking SD. 1994. Feeding ecology of fish, 416 pp. Academic Press, San Diego.

Giller P \& L Greenberg. 2015. The relationship between individual habitat use and diet in brown trout. Freshwater Biology 60:256-266.

González-Sansón G \& L Rodríguez-Viñas. 1983. Alimentación natural de Eugerres brasilianus (Cuvier) y Gerres cinereus (Wa1baum) (Pisces: Gerreidae) en las lagunas costeras de Tunas de Zaza, Cuba. Revista de Investigaciones Marinas, Cuba 4: 91-94.

Gosline WA. 1981. The evolution of the premaxillary protusion system in some teleostean fish groups. Journal of Zoology, London 193: 11-23.

Guevara E, H Álvarez, M Mascaró, C Rosas \& AJ Sánchez. 2007. Hábitos alimenticios y ecología trófica del pez Lutjanus griseus (Pisces: Lutjanidae) asociado a la vegetación sumergida en la Laguna de Términos, Campeche, México. Revista de Biología Tropical 55: 989-1004.

Hoffman M. 1978. The use of Pielou's method to determine sample size in food studies. In: Fish food habits studies. Proceedings of the Second Pacific Northwest Technical Workshop, pp. 56-61. Washington Sea Gran Publications. University of Washington, Seattle.

Hyslop EJ. 1980. Stomach contents analysis: a review of methods and their application. Journal of Fish Biology 17: 411-429.

Jones KMM. 2002. Behavioural overlap in six Caribbean labrid species: intra- and interspecific similarities. Environmental Biology of Fishes 65: 71-81.

Kerschner BA, MS Peterson \& RG Gilmore Jr. 1985. Ecotopic and ontogenetic trophic variation in mojarras (Pisces: Gerreidae). Estuaries 8: 311-322.

Kobelkowsky A \& M Alemán-Rivero. 2000. Branquiocráneo de la mojarra de mar, Diapterus auratus Ranzani (Pisces: Gerreidae). Universidad y Ciencia, México 16: 19-26.

Krebs C. 1999. Ecological methodology, 620 pp. AddisonWelsey, Menlo Park.

Labropoulou M \& A Eleftheriou. 1997. The foraging ecology of two pairs of congeneric demersal fish species: importance of morphological characteristics in prey selection. Journal of Fish Biology 50: 324-340.

Lilly GR \& AM Fleming. 1981. Size relationships in the predation by Atlantic cod, Gadus morhua, on capelin, Mallotus villosus, and sand lance, Ammodytes dubius, in the Newfoundland area. NAFO Scientific Council Studies, Canada 1: 41-45.
Ludwig JA \& JF Reynolds. 1988. Statistical ecology: APrimer on methods and computing, $337 \mathrm{pp}$. John Wiley and Sons, New York.

Machias A \& M Labropoulou. 2002. Intra-specific variation in resource use by red mullet, Mullus barbatus. Estuarine, Coastal and Shelf Science 55: 565-578.

Molinero A \& R Flos. 1991. Influence of sex and age on the feeding habits of the common sole Solea solea. Marine Biology 111: 493-501.

Morin PJ. 2011. Community ecology, 407 pp. Wiley-Blackwell, Oxford.

Motta PJ. 1984. Mechanics and functions of jaw protrusion in teleost fishes: a review. Copeia 1: 1-18.

Odum WE \& EJ Heald. 1972. Trophic analysis of an estuarine mangrove community. Bulletin of Marine Science 22: 671738.

Pauly D. 1986. A simple method for estimating the food consumption of fish populations from growth data and food conversion experiments. Fishery Bulletin 84: 827-842.

Ramos-Miranda J, L Quiniou, D Flores-Hernández, T DoChi, LA Ayala-Pérez \& A Sosa-López. 2005. Spatial and temporal changes in the nekton of Terminos Lagoon, Campeche, Mexico. Journal of Fish Biology 66: 513-530.

Randall JE. 1967. Food habits of reef fishes of the West Indies. Studies in Tropical Oceanography 5: 665-847.

Rivas A, E Méndez, A Torres \& L Martínez. 1999. Hábitos alimenticios de Eucinostomus gula y Eucinostomus argenteus (Pisces: Gerreidae), bahía de Mochima, Estado de Sucre, Venezuela. Boletín del Instituto Oceanográfico de Venezuela, Venezuela 38: 91-97.

Root RB. 1967. The niche exploitation pattern of the blue-grey gnatcatcher. Ecological Monographs 37: 317-350.

Ross ST. 1986. Resource partitioning in fish assemblages: A review of field studies. Copeia 2: 352-388.

Santos ACA \& FG Araújo. 1997. Hábitos alimentares de três espécies de Gerreidae (Osteichthyes, Perciformes) na Baía de Sepetiba, RJ. Brazilian Archives of Biology and Technology 40: 359-368.

Schaeffer B \& DE Rosen. 1961. Major adaptive levels in the evolution of the actinopterygian feeding mechanism. American Zoologist 1: 187-204.

Scharf FS, F Juanes \& RA Rountree. 2000. Predator sizeprey size relationships of marine fish predators: interspecific variation and effects of ontogeny and body size on trophicniche breadth. Marine Ecology Progress Series 208: 229248.

Schoener TW. 1974. Resource partitioning in ecological communities. Science 185: 27-39.

Scott S, R Pardo \& I Vila. 2007. Trophic niche overlap between two Chilean endemic species of Trichomycterus (Teleostei: Siluriformes). Revista Chilena de Historia Natural 80: 431437. 
Specziár A \& T Erõs. 2014. Dietary variability in shes: the roles of taxonomic, spatial, temporal and ontogenetic factors. Hydrobiologia 724: 109-125.

Stoner AW \& RJ Livingston. 1984. Ontogenetic patterns in diet and feeding morphology in sympatric sparid fishes from seagrass meadows. Copeia 1: 174-187.

Wootton RJ. 1990. Ecology of teleost fishes, 404 pp. Chapman and Hall, London.
Yáñez-Arancibia A \& JW Day. 1982. Ecological characterization of Terminos Lagoon, a tropical lagoonestuarine system in the southern Gulf of Mexico. Oceanologica Acta 5: 431-440.

Yáñez-Arancibia A \& P Sánchez-Gil. 1983. Environmental behavior of Campeche Sound ecological system, off Terminos Lagoon, México: Preliminary results. Anales del Instituto de Ciencias del Mar y Limnología, México 10: 117-136.

Recibido el 30 de junio de 2015 y aceptado el 4 de mayo de 2016

Editor: Claudia Bustos D. 